FACTA UNIVERSITATIS

Series: Mechanical Engineering Vol. 17, No 1, 2019, pp. 1 - 15

https://doi.org/10.22190/FUME190103008B

Original scientific paper

\title{
NUMERICAL METHODS FOR THE SIMULATION OF DEFORMATIONS AND STRESSES IN TURBINE BLADE FIR-TREE CONNECTIONS
}

\author{
Justus Benad \\ Berlin University of Technology, Berlin, Germany
}

\begin{abstract}
In this work, different numerical methods for simulating deformations and stresses in turbine blade fir-tree connections are examined. The main focus is on the Method of Dimensionality Reduction (MDR) and the Boundary Element Method (BEM). Generally, the fir-tree connections require a computationally expensive finite element setup. Their complex geometry exceeds the limitations of the faster numerical techniques which are used with great success within the framework of the half-space approximation. Ways of extending the application range of the MDR and the BEM to the particular problem of highly undulating surfaces of the fir-tree connection are shown and discussed.
\end{abstract}

Key Words: Fir-tree Connections, Navier Equation, Boundary Element Method, FFT

\section{INTRODUCTION}

The rotating components in a gas turbine are a challenge for both design and manufacturing. Especially turbine blades lead the way in terms of future technology [1]. Improvements of these components may result in lower weight, increased turbine performance, a longer life, and lower operating costs. For aero engines (see Fig. 1), such improvements have a positive impact on the entire aircraft $[2,3]$. This may lead to lower emissions and a reduced environmental impact. Among the most critical parts of the turbine are the fir-tree connections of turbine blade and turbine disk (see Fig. 2 and 3). The loads in these connections strongly influence the living of the blade and the disk. Indeed, turbine disks are among the components which are most prone to cracking in the entire engine [1]. Such a failure may cause severe damage to the aircraft (see Fig. 4). The resulting high safety requirements along with the afore mentioned high potential for future development and optimization of turbine blades, disks and their fir-tree connections lead to a demand for highly accurate and rapid simulation tools.

Received January 03, 2019 / Accepted March 11, 2019

Corresponding author: Justus Benad

Berlin University of Technology, Strasse des 17. Juni 135, 10623 Berlin, Germany

E-mail: mail@jbenad.com 
In this work, different numerical methods for simulating deformations and stresses in the turbine blade fir-tree connections are examined. The main focus is on the Method of Dimensionality Reduction (MDR) [4] and the FFT-based Boundary Element Method (BEM) [5]. Both methods have become standard tools in contact mechanics where they are applied with great success within the framework of the half-space approximation [6]. These rapid techniques are well-suited for demanding wear simulations (see for example [7], [8], and [9]).

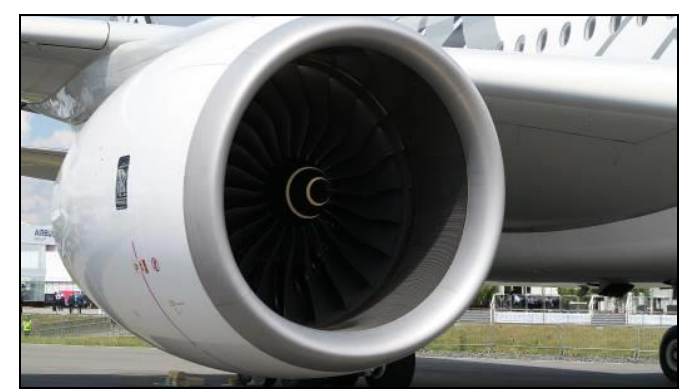

Fig. 1 Nacelle installation of the Rolls-Royce Trent $X W B$ turbofan engine on the Airbus A350-900 aircraft. Image: BENAD

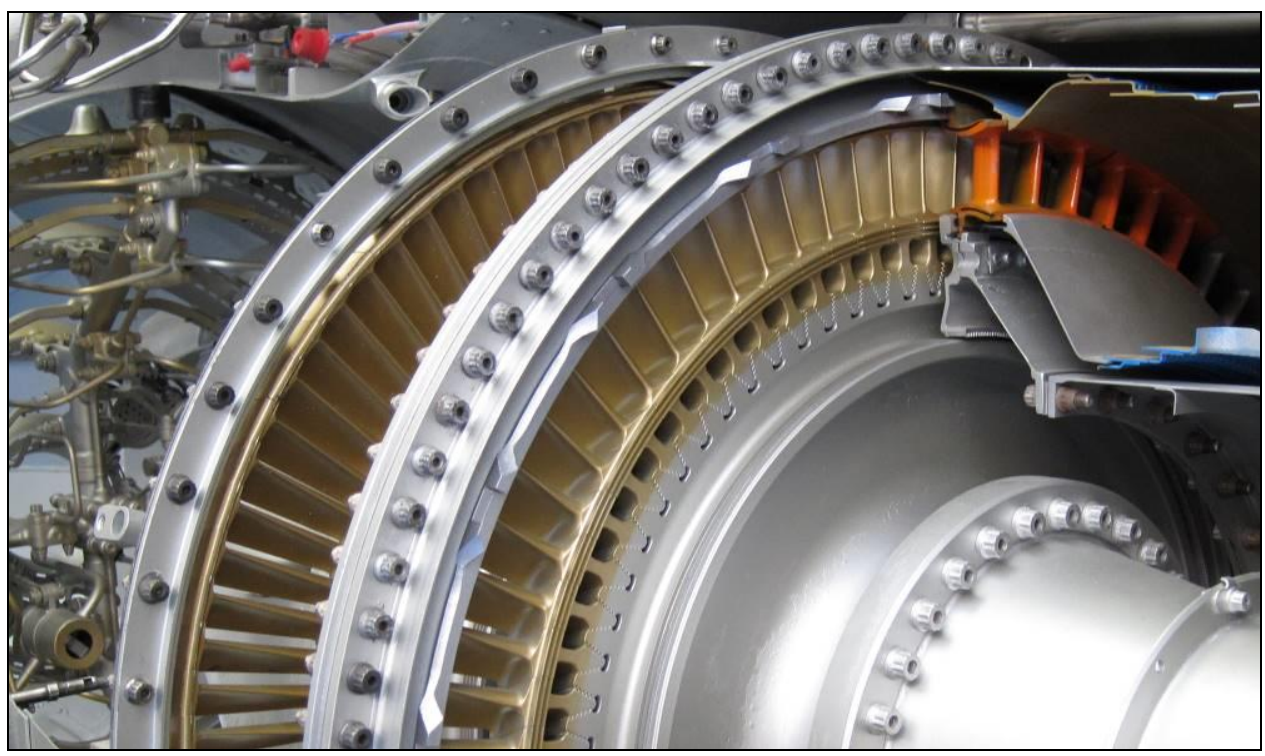

Fig. 2 Fir-tree connections of turbine blades (gold) and disk (silver) on a sectioned Rolls-Royce Turboméca Adour turbofan, an engine powering for example the McDonnell Douglas T-45 Goshawk aircraft. Image: CLEYNEN [10]

Fir-tree connections generally require a computationally expensive finite element setup. Their highly undulating surfaces and other complex geometrical elements in close proximity to the contact region make the application of the half-space theory a challenge. In this study, we closely examine the application range of the MDR and the FFT-based BEM beyond the 
half-space approximation. We also discuss ways of extending the methods so as to apply them to the particular problem of the complex geometry of the fir-tree connection.

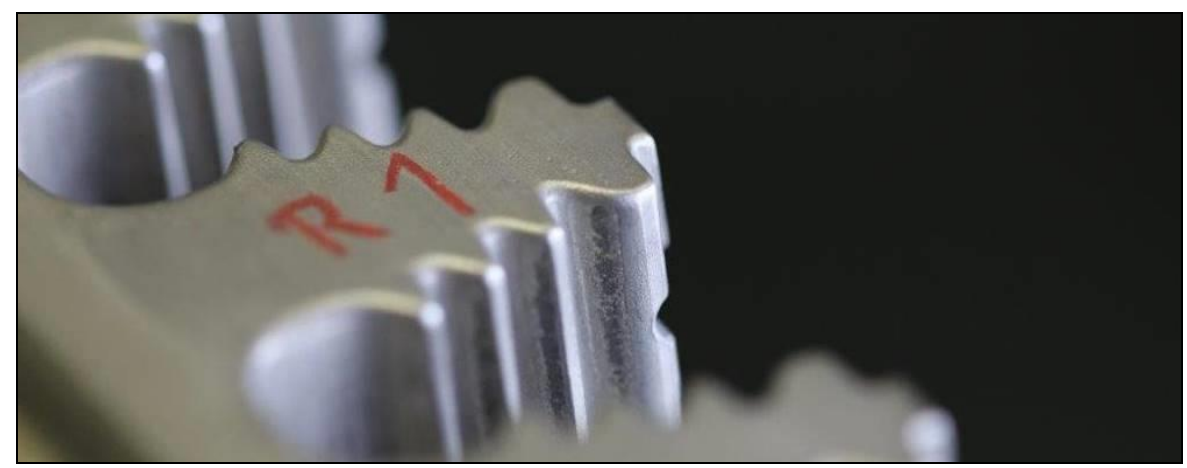

Fig. 3 A worn fir-tree connection on the disk of an aircraft turbine. Image used with the kind permission of Rolls-Royce

The parts of this work are organized as follows: we first discuss the MDR and show results of its application on a simplified fir-tree model. Likewise, the results of a finite element simulation of the same setup are shown. The findings of both methods are compared on a qualitative level. We then turn to the FFT-based BEM in its well-known and established form for the half-space, propose slight additions to the method and apply it to a fir-tree model. Again, the results are compared on a rough qualitative level to finite element results. In the last main section of this paper, we build on two recent studies [11] and [12], where the FFT-based BEM is performed for completely arbitrary shapes. We present exemplary results of this technique for the two-dimensional NAVIER equation. At the end of this work, the main findings are summarized in a conclusion.

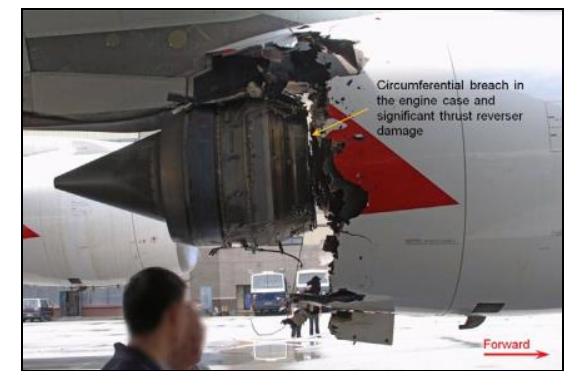

a)

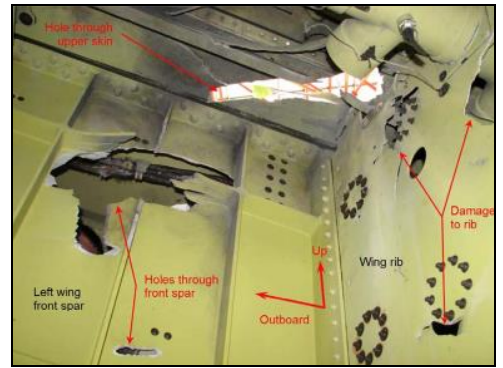

b)

Fig. 4 Example for the damage caused by an uncontained engine rotor failure. a) General damage to the engine, b) Example of internal damage to the left wing. Images: Australian Transport Safety Bureau, Safety Report, Aviation Occurrence Investigation: In-flight uncontained engine failure overhead Batam Island, Indonesia, 4. November 2010, Airbus A380-842 [13] 


\section{METHOD OF DIMENSIONALITY REDUCTION (MDR)}

The MDR is an efficient tool for calculating surface deformations and stresses of elastic or viscoelastic bodies which are brought into contact. The method was first proposed in 2007 [14] and has since developed to become a standard tool in contact mechanics [4], [15]. The technique is particularly easy to apply for axially-symmetric contacts and is generally used within the framework of the half-space approximation [6]. The MDR maps a three-dimensional contact to an equivalent contact of a transformed profile with a one-dimensional foundation of independent elastic or viscoelastic elements. From a numerical perspective, such a method is very appealing. First, this is due to a very low number of the degrees of freedom, and second, it is because the degrees of freedom are decoupled, which eliminates the need for iterations within the MDR domain. The transformations to the MDR domain and back can also be performed rapidly with an order of computational complexity which does not exceed the order of the number of discretization points of the one-dimensional profile. Therefore, the MDR is well suited for demanding wear simulations, which require an underlying highly efficient method to obtain the deformations and stresses. This procedure has been successfully implemented in the past, for example, for high-resolution simulations of gross slip wear [7], fretting-wear [16], and wear analysis of a heterogeneous material [8]. Fig. 5 shows a stage within a simple gross slip wear process of a parabolic rigid indenter with a given wear coefficient. This indenter is pressed into and moved across an elastic half-space. Also shown is the pressure distribution at the given stage. ARCHARD's law [17] is used as a wear model. In order to obtain a stage in the wear process such as the one displayed in Fig. 5, the required computational time is only a fraction of a second on a small personal computer when the MDR is used to calculate the deformations and stresses within the wear iteration.

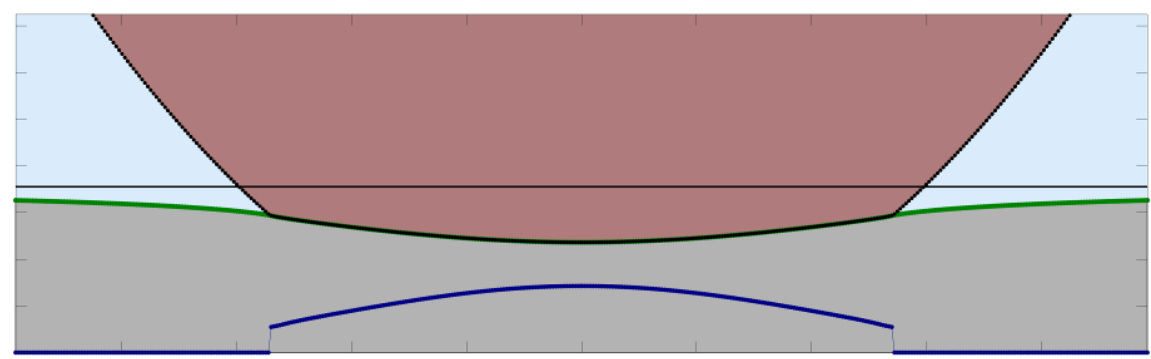

Fig. 5 An exemplary stage of a simple gross slip wear process of a parabolic rigid indenter with a given wear coefficient (light red upper shape enclosed with black dotted line) which is pressed into and moved across an elastic half-space (grey area with green dotted line at the surface, the thin horizontal black line marks the undisturbed surface). At the bottom of the image the form of the pressure distribution at the given stage is shown which is zero outside the contact area and takes positive values within the contact area (blue line with dots). The MDR was used to obtain the deformations and stresses while the ARCHARD's law was used to model the wear 
Given the efficiency of the MDR and the success when it comes to wear simulations, it would be desirable to apply the method for calculating deformations and stresses in turbine blade fir-tree connections. Fig. 6a shows a schematic view of one tooth of a turbine blade in contact with its counterpart on the disc. A close-up view, which is also stretched in the $z$-direction, is displayed in Fig. 6 b.

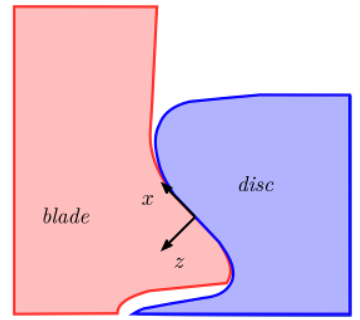

a)

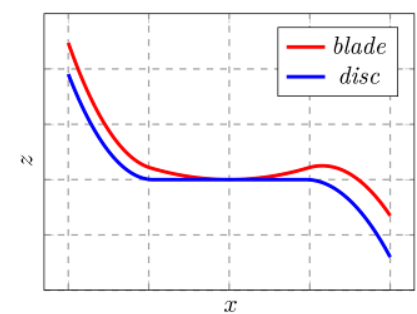

b)

Fig. 6 a) Schematic view of one tooth of a turbine blade in contact with its counterpart on the disk, b) close up view of the contacting profiles stretched in $z$-direction. Images: DIERCKS (modified to fit the context). Images used with the kind permission of Rolls-Royce

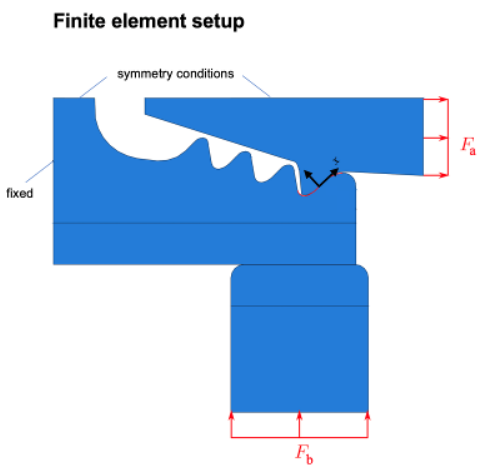

a)

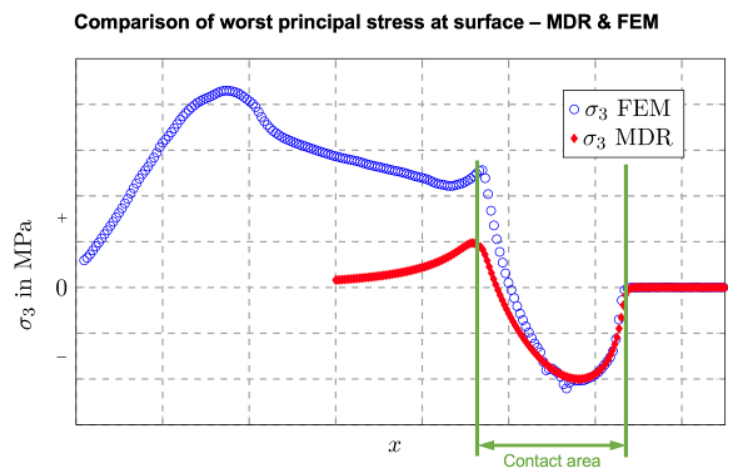

b)

Fig. 7 a) Finite element setup for the calculation of deformations and stresses on a simplified fir-tree model with only one tooth in contact, b) exemplary results of the finite element model (blue) and the MDR approximation (red) for the worst principal stress at the disk surface (marked with a thin red line in the left graph a). Images: DIERCKS (modified to fit the context). Images used with the kind permission of Rolls-Royce

In a rough first approximation, the problem can be regarded as a simple two-dimensional contact of cylindrical lying indenter with a half-space. The transformed profile to be used in the MDR for such a line contact can be obtained directly [6] (see also [18]), or one can make use of FABRIKANT's approximation (see [19]) to scale a corresponding rotationally symmetric solution to the real contact area as a rough first estimate. The latter approach was adopted here. With the resulting normal and tangential loads in the contact area, one can then obtain all 
components of the stress tensor at the surface using the fundamental solutions of BOUSSINESQ and CERRUTI (see [20]), which makes it possible to display the worst principle stress at the surface. Exemplary results for the worst principle stress at the surface obtained in such a fashion are displayed in Fig. $7 \mathrm{~b}$ along with finite element results for comparison. The finite element setup which was used is displayed in Fig. 7a. Note that for simplicity a blade root with only one tooth was considered in this first model.

It transpires that, at least in the contact area, this very crude MDR approach already delivers the results which agree to a certain extent with those obtained with the more appropriate finite element model. The most striking difference is, of course, attained as the bottom of the first lobe is approached (region left of the contact area in Fig. 7b). The influence of this notch is not modeled with the half-space approach. In the following section, we will remain within the framework of the half-space theory as we are interested primarily in the properties in the contact area. However, we shall still turn to a less confined model, the Boundary Element Method. The setup will remain the same, with the exception that all four teeth of the fir-tree will be considered.

\section{FFT-BASED BOUNDARY ELEMENT METHOD (BEM) FOR THE HALF-SPACE}

The Boundary Element Method (BEM) is used in many engineering applications. For some special cases, such as the simulation of tribological contacts where the technique is applied with the half-space approximation, the tool has set new standards in recent years, becoming the method of choice both in academic and industrial research and development [15]. The integral equations of the BEM simplify to convolutions when the boundary can be approximated as a half-space surface. As such, the integrals can easily be obtained with the Fast-Fourier Transformation (FFT). The computational complexity to obtain the deformations and stresses at the half-space surface is $O\left(n^{2} \log n\right)$ for a surface with $n \times n$ discretization points. Therein lies the great advantage of using the FFT-based half-space BEM for when the FFT is not used and the entire matrix of the linear system is built, the complexity of the BEM is $O\left(n^{4}\right)$. Therefore, we will for now retain the assumption of a half-space, in order to apply this well-known, established and efficient FFT-based half-space BEM to the contact problem of the fir-tree connection.

The two-dimensional convolution one obtains for the half-space is

$$
u_{a}(x, y)=\int_{S} K_{a b}\left(x-x_{0}, y-y_{0}\right) \sigma_{b} d x_{0} d y_{0}
$$

where $u$ is the deformation, $\sigma$ is the load, $a$ is the direction of the deformation and $b$ is the direction of the load, $(a, b) \in\{x, y, z\}$ [21]. Typically, the half-space approximation is used only when the gradients of the surfaces in the contact region are very small. This is not the case for the fir-tree. However, the error which is made can be kept at bay by careful consideration of the surface constraints such as geometry and friction of the actual curved surfaces.

Consider a fir-tree connection and coordinate system as displayed in Fig. 8. Unlike in the previous section where the half-space was aligned with the contacting surfaces of the single lobe, the half-space is now placed along the $X-Y$ plane in Fig. 8 to run through the entire fir-tree connection. The half-space surface can then be imagined to be perturbed from its original flat state to the highly undulating shape in the $Z$-direction. As a first step, it 
shall also be assumed that one of the contact partners is elastic and the other is rigid. This is a common approach in many problems in contact mechanics. Once the solution is obtained it can generally be transformed back to the original problem with the knowledge of the elastic properties of the contacting bodies [6], [22]. The disk shall be modeled as an elastic half-space, and the blade shall be modeled as a rigid body in the following.

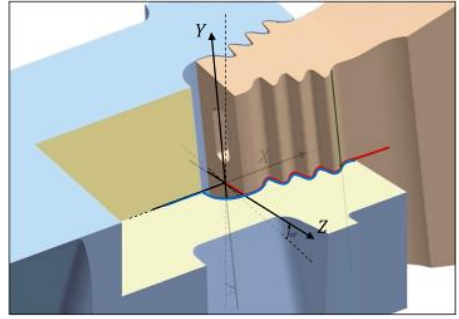

a)

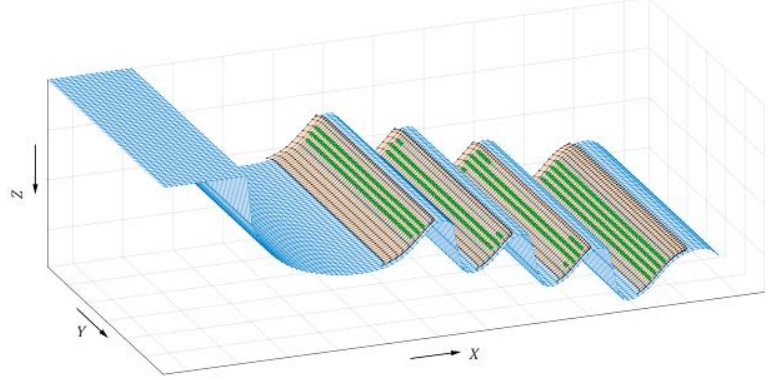

b)

Fig. 8 a) Geometry of a fir-tree connection of a blade model (light brown) and a disk model (light blue). A rectangular block is cut out of the disk model (sections are displayed yellow) to reveal the global coordinate system and the 2D curves (blue and red). b) Extrusion along the $Y$-axis of the 2D curves and contact area (green markers) for an exemplary indentation in $X$-direction. The disk is modeled as an elastic half-space and the turbine blade as a rigid indenter. The black discretization points of the turbine blade surface are given by a linear interpolation of the disk surface discretization points onto the turbine blade surface along the $X$-axis. For visualization purposes the extrusion width shown here is small and only a rough discretization is displayed. The beginning and end of the extrusion of the turbine blade profile are slightly rounded of in the direction of lower $X$ to ensure a smooth indentation at these edges. Image used with the kind permission of Rolls-Royce

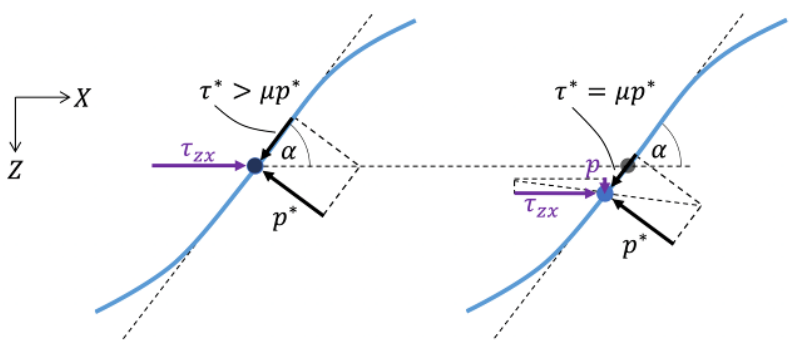

Fig. 9 Left: Components of a shear stress $\tau_{z x}$ relative to the curved surface. $\tau^{*}$ cannot be maintained because (3) is not fulfilled. Right: The grid point of the disk (blue) has now slid down along the rigid profile to its equilibrium position where $\tau^{*}=\mu p^{*}$. This results in a lower $\tau_{z x}$ and a new component $p$ relative to the even half-space surface. Image used with the kind permission of Rolls-Royce. 
Consider now a single grid point on the disk profile. For some indentation, that is the displacement of the blade in the $X$-direction from its position of first contact, the point on the disk is also deflected in the direction of the $X$-axis by a small distance $u_{x}$. As the disk profile is modeled as an elastic half-space it can be regarded as even for the determination of the dependencies of surface deformations and forces. Under the assumption that the normal and tangential problem are decoupled at the even half-space surface [21] the deflection in the direction of the $X$-axis is caused by a shear stress $\tau_{z x}$ at the grid point. However, in order to model the friction conditions, the components of the stress at the grid point relative to the actual curved surface

$$
\begin{gathered}
\tau^{*}=\tau_{z x} \cos \alpha-p \sin \alpha, \\
p^{*}=\tau_{z x} \sin \alpha+p \cos \alpha .
\end{gathered}
$$

need to be considered. This is illustrated in Fig. 9. Assuming there exists friction according to the COULOMB's law of friction in its simplest form [23], it has to be

$$
\tau^{*} \leq \mu p^{*}
$$

so that the equilibrium can be maintained. Otherwise, the point will slide. Whether the condition (3) is fulfilled depends only on the geometry and the friction coefficient. In the left graph in Fig. 9 the condition is not fulfilled. Thus, the grid point of the disk profile slides until the relation (3) is fulfilled again (right graph in Fig. 9). The corresponding new values for $\tau^{*}$ and $p^{*}$ cause a change of the stresses in the reference system of the even half-space which can be determined with the reverse transformation

$$
\begin{aligned}
\tau_{z x} & =p^{*} \sin \alpha+\tau^{*} \cos \alpha, \\
p & =p^{*} \cos \alpha-\tau^{*} \sin \alpha .
\end{aligned}
$$

Fig. 8b displays results which are obtained in such a fashion. The contact area (green dots) is shown for an exemplary indentation. The inner values for the displacements and stresses can be obtained from the conditions at the surface with the fundamental solutions of BOUSSINESQ and CERRUTI (see [20]) as in Section 2. Fig. 10 displays a qualitative view of the worst principal stress in the disk (upper image). For comparison, the finite element results are displayed below.

As in the previous section, it transpires that although there is some rough agreement of the results, there are also quite substantial differences, even on the qualitative level. The most striking difference is once more the notch stress in the lower regions of the lobes. The maximum is clearly shifted towards the contact area for the half-space approach, while it is at a much lower position of the lobes for the finite element results which consider the actual shape more appropriately. While the half-space approach may deliver sufficient results in the contact regions for the analysis of particular problems, the overall results still remain unsatisfactory. Therefore, other methods to utilize the efficiency of the convolution for the calculation of deformations and stresses in complex shapes such as the fir-tree must be found. One such approach is described in detail in the next section. 


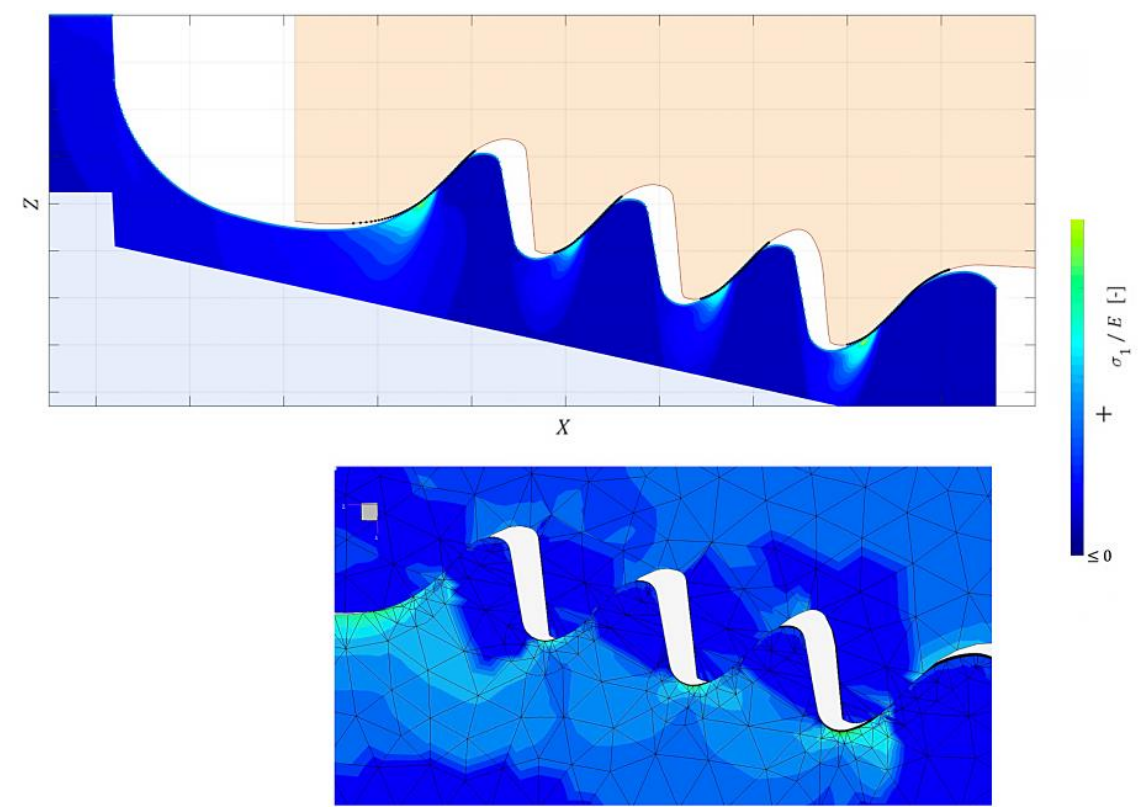

Fig. 10 A rough qualitative comparison of the results for the worst principal stress as obtained with the FFT-based BEM for the half-space (upper image) and a finite element simulation (lower image). The maximum of the worst principle stress is attained in the lower notch regions in the FE approach, while for the half-space approach it is shifted upwards to the lower ends of the contact regions. Image used with the kind permission of Rolls-Royce

\section{FFT-BASED BEM BEYOND THE HALF-SPACE APPROXIMATION}

It is shown in the last two sections that the rapid half-space approach may provide a rough approximation of the results in the contact regions of a fir-tree; yet, the overall results were unsatisfactory. Therefore, other methods must be found in order to utilize the efficiency of the convolution for calculating deformations and stresses in complex shapes. Various techniques which have been developed in the past may be applied to accomplish this task. In 1991, GAO introduced a first-order perturbation method for the half-space approximation to take into account stress concentration effects of slightly undulating surfaces [24]. In later years, various methods were developed to accelerate the classical BEM for completely arbitrary shapes which, in part, make use of the low computational complexity of the FFT (see for example [25], [26] and [27]), or utilize other techniques such as hierarchical matrices (see for example [28]) to accelerate the calculation. Recently, it was illustrated in [11] and [12] that the integral equations of the BEM for completely arbitrary shapes (no half-space) can be obtained in a manner very similar to the FFT-based half-space approach: For the case of the half-space, the boundary integral (1) is evaluated in the plane of the two coordinates $x$ and $y$ which perfectly aligns with the even half-space surface. This makes it possible to align a regular two-dimensional grid on which the FFT is performed with the domain (see Fig. 11a). 


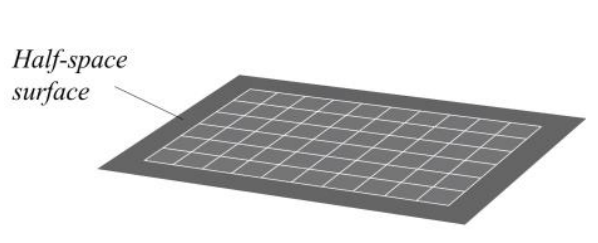

a)

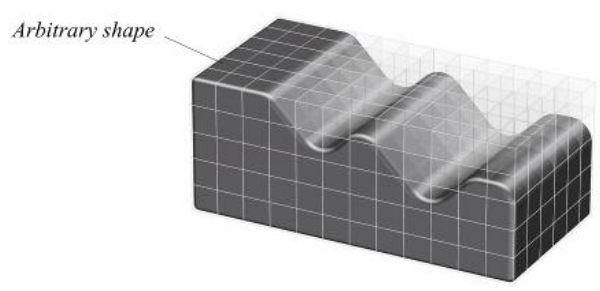

b)

Fig. 11 a) A uniform grid aligned with the even surface of a half-space, b) an arbitrary shape fully enclosed with a uniform three-dimensional grid

For arbitrary shapes, the boundary integral equations represent convolutions over the entire three-dimensional space which encloses the arbitrary shape (see Fig. 11b). It is shown in [11] and [12] how one has to appropriately set zeros at grid points which are not in close proximity to the actual surface so as not to distort the results one obtains with this technique. Using the FFT in such a fashion as to obtain the boundary integrals on arbitrary shapes lowers the computational complexity from $O\left(n^{4}\right)$ to $O\left(n^{3} \log n^{1.5}\right)$. While this is still for one dimension higher than the $O\left(n^{2} \log n\right)$ complexity of the half space, the technique opens up opportunities for further reduction in computational complexity through a variable grid (see [12]), and offers the advantage of utilizing highly efficient implementation of the FFT, which can be accelerated even further on parallel systems such as the Graphics Processing Unit (GPU).

We now build upon the results found in [11] and [12] where the FFT is used to accelerate the calculation of the boundary integrals on arbitrary shapes for the LAPLACE equation. The same technique is applied here, but for the two-dimensional NAVIER equation.

\section{$\underline{\text { Boundary integrals }}$}

NAVIER's equation is

$$
\frac{1}{1-2 v} u_{j, j i}+u_{i, j j}+\frac{1}{\mu} b_{i}=0
$$

where $i, j \in\{1,2,3\}$ and $b_{i}$ is the force density field [20]. For the case of plane displacements, the NAVIER equation remains as in (5), but with $i, j \in\{1,2\}$, [29]. This case can then easily be transformed into the case of plane stress, by replacing $v$ with $v /(1+v)$ and leaving the value for $\mu$ unchanged [29], see also [30] and [31].

A single unit point force shall act in a point $\underline{x}_{0}$, and in the direction of a unit vector $\underline{e}$, so that in Eq. (5), it is $b_{i}=\delta\left(\underline{x}-\underline{x}_{0}\right) e_{i}$, which yields the equation

$$
\frac{1}{1-2 v} u_{j, j i}^{*}+u_{i, j j}^{*}=-\frac{1}{\mu} \delta\left(\underline{x}-\underline{x}_{0}\right) e_{i} .
$$

The solution $u_{i}^{*}$ of Eq. (6) is called the fundamental solution of Eq. (5). It can be expressed in terms of a matrix vector product with

$$
u_{i}^{*}=e_{k} u_{k i}^{*} .
$$


With the BETTI's theorem [32], the divergence theorem, and the definition of the fundamental solution given in Eq. (6), one obtains (see [33]) SoMIGLIANA's identity

$$
u_{i}\left(\underline{x}_{0}\right)=\int_{S} u_{i j}^{*}\left(\underline{x}, \underline{x}_{0}\right) t_{j}(\underline{x}) d S-\int_{S} t_{i j}^{*}\left(\underline{x}, \underline{x}_{0}\right) u_{j}(\underline{x}) d S,
$$

which relates the deformations $u_{j}(\underline{x})$ and stress vector $t_{j}(\underline{x})=\sigma_{j k}(\underline{x}) n_{k}(\underline{x})$ on the boundary $S$ (outward normal vector $n_{k}$ ) to the deformation $u_{j}\left(\underline{x}_{0}\right)$ at a particular inner point $\underline{x}_{0}$. The term $t_{i j}^{*}\left(\underline{x}, \underline{x}_{0}\right)$ which occurs in (8) is briefly explained in the following: note that a certain stress field $\sigma_{i j}{ }^{*}$ belongs to the fundamental solution for the deformation field $u_{i}{ }^{*}$. Depending on the position $\underline{x}_{0}$, this sets a corresponding stress vector $t_{j}^{*}\left(\underline{x}, \underline{x}_{0}\right)=\sigma_{j k}^{*}\left(\underline{x}, \underline{x}_{0}\right) n_{k}(\underline{x})$ on the given boundary. A matrix $t_{i j}{ }^{*}$ is then constructed so as to express this stress vector with

$$
t_{j}^{*}=e_{i} t_{i j}^{*} .
$$

Accordingly, and with the material law for a linearly elastic isotropic solid [34], one obtains

$$
t_{i j}^{*}=\frac{2 v \mu}{1-2 v} u_{i k, k}^{*} n_{j}+\mu\left(u_{i j, k}^{*} n_{k}+u_{i k, j}^{*} n_{k}\right) .
$$

The fundamental solution for the arbitrary three-dimensional case or for the cases of plane displacements or plane stress can be found in the literature. For simplicity, we will from now on only consider the case of plane displacements. Through Eq. (7), the two-dimensional fundamental solution of Eq. (5) is for this case given with [33]

$$
u_{i j}^{*}=\frac{-(3-4 v) \ln (r) \delta_{i j}+\left(x_{i}-x_{0 i}\right)\left(x_{j}-x_{0 j}\right) / r^{2}}{8 \pi \mu(1-v)},
$$

where $r=\sqrt{\left(x-x_{0}\right)^{2}+\left(y-y_{0}\right)^{2}}$. Note that we choose to denote $x_{i=1}$ as $x$ and $x_{i=2}$ as $y$. Inserting Eq. (11) into Eq. (10) yields

$$
\begin{aligned}
t_{i j}^{*}=-\frac{1}{4 \pi(1-v) r} & \left((1-2 v) \delta_{i j}+2 \frac{\left(x_{i}-x_{0 i}\right)\left(x_{j}-x_{0 j}\right)}{r^{2}}\right) \frac{x_{k}-x_{0 k}}{r} n_{k} \\
& \left.+(1-2 v)\left(\frac{x_{j}-x_{0 j}}{r} n_{i}-\frac{x_{i}-x_{0 i}}{r} n_{j}\right)\right)
\end{aligned}
$$

providing through Eq. (9) the stress vector $t_{j}^{*}$ of the fundamental solution on the boundary for a certain $\underline{x}_{0}$.

Let us now insert the fundamental solution, that is Eqs. (11) and (12), into SOMIGLIANA's identity, Eq. (8). When we write out the result in detail and choose to denote $u_{i=1}=u$ and $u_{i=2}=v$, we obtain for the first component of the deformations at a particular inner point $\underline{x}_{0}=\left(x_{0}, y_{0}\right)$ due to the deformations and stresses at the surface 


$$
\begin{gathered}
u\left(x_{0}, y_{0}\right)=\frac{1}{8 \pi \mu(1-v)} \cdot \int_{S}-(3-4 v) \ln (r) t_{1}+\frac{t_{1}\left(x-x_{0}\right)^{2}}{r^{2}}+\frac{t_{2}\left(x-x_{0}\right)\left(y-y_{0}\right)}{r^{2}} d S \\
-\frac{1}{4 \pi(1-v)} \cdot\left[\int_{S}\left(1-2 v+2 \frac{\left(x-x_{0}\right)^{2}}{r^{2}}\right)\left(n_{1} \frac{u\left(x-x_{0}\right)}{r^{2}}+n_{2} \frac{u\left(y-y_{0}\right)}{r^{2}}\right) d S\right. \\
+\int_{S} \frac{2\left(x-x_{0}\right)\left(y-y_{0}\right)}{r^{4}} v\left(n_{1}\left(x-x_{0}\right)+n_{2}\left(y-y_{0}\right)\right) \\
\left.+\frac{1-2 v}{r^{2}} v\left(n_{1}\left(y-y_{0}\right)-n_{2}\left(x-x_{0}\right)\right) d S\right] .
\end{gathered}
$$

For the second component, we obtain

$$
\begin{gathered}
v\left(x_{0}, y_{0}\right)=\frac{1}{8 \pi \mu(1-v)} \cdot \int_{S}-(3-4 v) \ln (r) t_{2}+\frac{t_{2}\left(y-y_{0}\right)^{2}}{r^{2}}+\frac{t_{1}\left(x-x_{0}\right)\left(y-y_{0}\right)}{r^{2}} d S \\
-\frac{1}{4 \pi(1-v)} \cdot\left[\int_{S}\left(1-2 v+2 \frac{\left(y-y_{0}\right)^{2}}{r^{2}}\right)\left(n_{1} \frac{v\left(x-x_{0}\right)}{r^{2}}+n_{2} \frac{v\left(y-y_{0}\right)}{r^{2}}\right) d S\right. \\
+\int_{S} \frac{2\left(x-x_{0}\right)\left(y-y_{0}\right)}{r^{4}} u\left(n_{1}\left(x-x_{0}\right)+n_{2}\left(y-y_{0}\right)\right) \\
\left.+\frac{1-2 v}{r^{2}} u\left(n_{2}\left(x-x_{0}\right)-n_{1}\left(y-y_{0}\right)\right) d S\right] .
\end{gathered}
$$

\section{Exemplary results}

We now seek to perform an exemplary calculation of the boundary integrals (13) and (14) for an arbitrary shape with the FFT making use of the use of technique [12]. In order to test the method, we use a simple analytical solution for the NAVIER equation

$$
u=\frac{1-v}{2 \mu} \sigma_{0} x+C_{1}, \quad v=-\frac{v}{2 \mu} \sigma_{0} y+C_{2}
$$

with the stress distribution

$$
\sigma_{x}=\sigma_{0}, \quad \sigma_{y}=0, \quad \sigma_{x y}=0
$$

The chosen geometry of the shape and Eqs. (15) and (16) then set the analytical solutions for the boundary values for the deformations and the stress vector. Both the chosen shape and the stress vector on its boundary are displayed in Fig. 12a. The raw FFT results for the deformations $u$ and $v$, as obtained for the chosen numerical values of $\mu=1, v=0.3$ and $\sigma_{0}=2$, are displayed with colored surfaces in Fig. $12 \mathrm{~b}$. The corresponding analytical solution for the deformation on the boundary is displayed with the red line in Fig. 12b. The numerical results clearly show the linear dependence we expect (see Eq. (15)). Very close to the boundary there are slight oscillations in the raw results. Such small distortions were observed on other problems in previous studies and can generally be eliminated easily without an increase in computational complexity (see [12]). We can conclude from the small example that the FFT approach commonly used in contact mechanics to solve a BEM 
problem for a half-space may be adopted in a similar way to solve problems on completely arbitrary shapes. However, we have presented only a first rough example and extensive research is required to develop the method further.

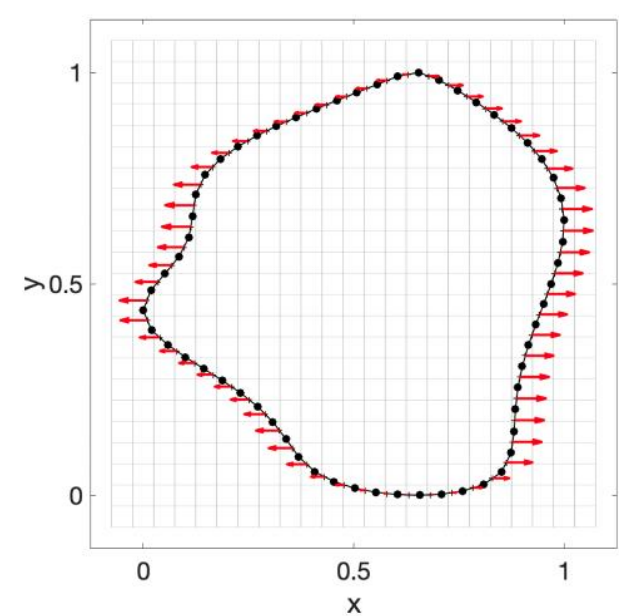

a)

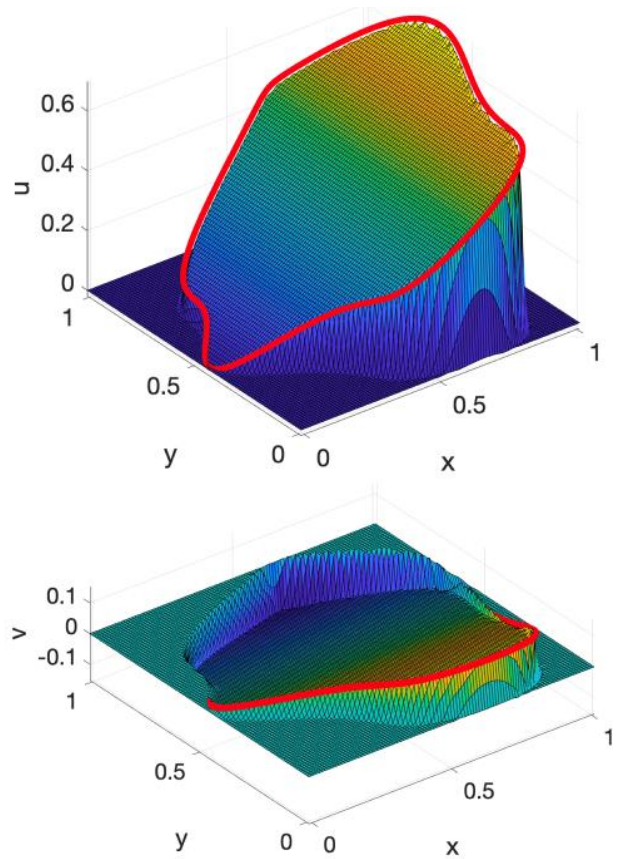

b)

Fig. 12 a) An arbitrary two-dimensional shape discretized with boundary elements and fully enclosed with a two-dimensional grid for the application of the FFT. The chosen stress vector for the test calculation is displayed with red arrows. The resolution of both the boundary and the FFT gird is lower than in the adjacent images merely for purposes of a better visualization. b) Raw results of the convolution for the deformations $u$ and $v$ on the boundary of the chosen shape as obtained for numerical values of $\mu=1, \nu=0.3$ and $\sigma_{0}=2$. The corresponding analytical solution for the deformation on the boundary is displayed with a red line

\section{CONCLUSION}

In this paper, different numerical methods for simulating deformations and stresses in the turbine blade fir-tree connections were discussed and in part extended for the application on the complex geometry of the fir-tree. It was highlighted that both the Method of Dimensionality Reduction (MDR) and the Boundary Element Method (BEM) are rapid simulation techniques for the half-space and are well suited for wear simulations. It was shown that both techniques can provide first insights to better understand the fir-tree. In the region of the contact area of the fir-tree lobes, the results for deformations and stresses obtained with the half-space models are similar to those obtained with finite 
element models. Generally, however, the complex fir-tree geometry exceeds the limitations of the half-space models. Also mentioned are ways of extending the half-space model to the geometry of the fir-tree connection, such as a first-order perturbation method for the half-space approximation to take into account stress concentration effects at slightly undulating surfaces introduced by GAO in 1991 [24]. In addition, a recent approach for completely arbitrary shapes (see [11] and [12]) inspired by the rapid FFT based half-space BEM, was discussed and applied on the NAVIER equation. A small example was presented to indicate that the use of this technique which utilizes the FFT to obtain the boundary integrals on completely arbitrary shapes may be a viable method and worthy of further investigation. This is due to the lower computational complexity of the method $O\left(n^{3} \log n^{1.5}\right)$ than the inversion of the standard BEM matrix $O\left(n^{4}\right)$. Other aspects which make the technique appealing are the potential of decreasing this complexity even further through adaptive grids, efficient parallel implementations of the FFT, and the similarity of the method to the established and very successful FFT-base BEM for the half-space.

Acknowledgements: The author would like to thank V. L. Popov, A. Golowin, A. Lacher, P. Duó, and $P$. Diercks for many valuable discussions on the topic. The author is particularly grateful for the kind permission of Rolls-Royce to use illustrative images throughout this work.

\section{REFERENCES}

1. Bräunling, W., 2015, Flugzeugtriebwerke, 3 ed, Springer, Berlin.

2. Torenbeek, E., 1982, Synthesis of Subsonic Airplane Design, Kluwer Academic Publishers, Dordrecht.

3. Raymer, D., 1999, Aircraft Design: A Conceptual Approach, 3 ed, American Institute of Aeronautics and Astronautics, Inc., Reston.

4. Popov, V.L., Heß, M., 2015, Method of dimensionality reduction in contact mechanics and friction, Springer, Berlin.

5. Putignano, C., Afferrante, L., Carbone, G., Demelio, G., 2012, A new efficient numerical method for contact mechanics of rough surfaces, International Journal of Solids and Structures, 49(2), pp. 338-343.

6. Popov, V.L., 2017, Contact Mechanics and Friction, 2 ed, Springer, Berlin.

7. Dimaki, A., Dmitriev, A., Menga, N., Papangelo, A., Ciavarella, M., Popov, V.L., 2016, Fast high-resolution simulation of the gross slip wear of axially symmetric contacts, Tribology Transactions, 59(1), pp. 189-194.

8. Li, Q., Forsbach, F., Schuster, M., Pielsticker, D., Popov, V.L., 2018, Wear Analysis of a Heterogeneous Annular Cylinder, Lubricants, 6(1), 28

9. Popov, V.L., Pohrt, R., 2018, Adhesive wear and particle emission: Numerical approach based on asperity-free formulation of Rabinowicz criterion, Friction, 6(3), pp. 260-273.

10. Cleynen, O., 2012, Turbine of a sectioned Rolls-Royce Turboméca Adour turbofan, accessed 2019 at https://upload.wikimedia.org/wikipedia/commons/7/7e/Turbine_of_a_sectioned_Rolls-Royce_Turbom\% C3\%A9ca_Adour_turbofan.jpg.

11. Benad, J., 2018, Acceleration of the Boundary Element Method for arbitrary shapes with the Fast Fourier Transformation, arXiv preprint, arXiv:1809.00845.

12. Benad, J., 2018, Efficient calculation of the BEM integrals on arbitrary shapes with the FFT, Facta Universitatis-Series Mechanical Engineering, 16(3), pp. 405-417

13. ATSB Transport Safety Report, 2013, In-flight uncontained engine failure Airbus A380-842, accessed 2019 at https://www.atsb.gov.au/publications/investigation_reports/2010/aair/ao-2010-089.aspx.

14. Popov, V.L., Psakhie, S., 2007, Numerical simulation methods in tribology, Tribology International, 40(6), pp. 916-923.

15. Popov, V.L., 2018, Is tribology approaching its Golden Age? Grand Challenges in Engineering Education and Tribological Research, Frontiers in Mechanical Engineering, 4, pp. 16.

16. Dimaki, A., Dmitriev, A., Chai, Y., Popov, V.L., 2014, Rapid simulation procedure for fretting wear on the basis of the method of dimensionality reduction, International Journal of Solids and Structures, 51(25-26), pp. 4215-4220. 
17. Archard, J., Hirst, W., 1956, The wear of metals under unlubricated conditions, Proc. R. Soc. Lond. A, 236(1206), pp. 397-410.

18. Nakano, K., Kawaguchi, K., Takeshima, K., Shiraishi, Y., Forsbach, F., Benad, J., Popov, M., Popov, V.L., 2019, Investigation on dynamic response of rubber in frictional contact, Frontiers in Mechanical Engineering, 5, pp. 9.

19. Barber, J., 2018, Contact Mechanics, Solid Mechanics and Its Applications, Springer, New York.

20. Hahn, H., 1985, Elastizitätstheorie, Vieweg Teubner Verlag, Wiesbaden.

21. Pohrt, R., Li, Q., 2014, Complete Boundary Element Formulation for Normal and Tangential Contact Problems, Physical Mesomechanics, 17(4), pp. 334-340.

22. Johnson, K., 2003, Contact mechanics, Cambridge University Press.

23. Coulomb, C., 1821, Theorie des machines simple, Bachelier, Paris.

24. Gao, H., 1991, Stress concentration at slightly undulating surfaces, Journal of the Mechanics and Physics of Solids, 39(4), pp. 443-458.

25. Phillips, J., White, K., 1997, A precorrected FFT-method for electrostatic analysis of complicated 3-D structues, IEEE Transactions on Computer-Aided Design of Integrated Circuits and Systems, 16(10), pp. 1059-1072.

26. Masters, N., Ye, W., 2004, Fast BEM solution for coupled electrostatic and linear elastic problems, NSTI-Nanotech, 2, pp. 426-429.

27. Lim, K., He, X., Lim, S., 2008, Fast Fourier transform on multipoles (FFTM) algorithm for Laplace equation with direct and indirect boundary element method, Computational Mechanics, 41, pp. 313-323.

28. Benedetti, I., Aliabadi, M., Davi, G., 2008, A fast 3D dual boundary element method based on hierarchical matrices, International journal of solids and structures, 45(7-8), pp. 2355-2376.

29. Irgens, F., 2008, Theory of Elasticity, Continuum Mechanics, Springer, Berlin.

30. Galin, L., 2008, Plane Elasticity Theory, Contact Problems, G. Gladwell, Editor, Springer, Dordrecht.

31. Kelly, P., 2013, Linear Elasticity, An introduction to Solid Mechanics (Lecture Notes), University of Auckland.

32. Betti, E., 1872, Teoria della elasticità, Il Nuovo Cimento, 7(1), pp. 69-97.

33. Gaul, L., Fiedler, C., 2013, Methode der Randelemente in Statik und Dynamik, 2 ed, Springer, Berlin.

34. Gross, D., Hauger, W., Wriggers, P., 2014, Technische Mechanik 4, 9 ed, Springer, Berlin. 